\title{
Simulations of energy dissipation and non-thermal desorption on amorphous solid water
}

\author{
H. M. Cuppen(i) and A. Fredon \\ Radboud University, Institute for Molecules and Materials, Nijmegen, the Netherlands \\ email: h.cuppen@science.ru.nl
}

\begin{abstract}
Dust particles covered by icy mantles play a crucial role in the formation of molecules in the Interstellar Medium (ISM). These icy mantles are mainly composed of water but many other chemical species are also contained in these ices. These compounds can diffuse and meet each other to react. It is through these surface reactions that new saturated species are formed. Photodissociation reactions are also thought to play a crucial role in the formation of radical species. Complex organic molecules are formed through an intricated network of photodissociation and surface reactions.

Both type of reactions release energy. Surface reactions are typically exothermic by a few eV, whereas photodissociation reactions are triggered by the absorption of a UV photon, resulting in the formation of highly excited products. The excited reaction products can apply this energy for desorption or diffusion, making products more mobile than predicted when considering only thermal hopping. The energy could further lead to annealing or deformation of the ice structure.

Here we would like to quantify the relative importance of these different energy dissipation routes. For this we performed thousands of Molecular Dynamics simulations for three different species $\left(\mathrm{CO}_{2}, \mathrm{H}_{2} \mathrm{O}\right.$ and $\left.\mathrm{CH}_{4}\right)$ on top of a water ice surface. We consider different types of excitation such as translational, rotational, and/or vibrational excitation. The applied substrate is an amorphous solid water surface (ASW).
\end{abstract}

Keywords. astrochemistry, molecular processes, methods: numerical, ISM: molecules

\section{Introduction}

Close to 200 molecules have been identified in interstellar space (see http://www. astro.uni-koeln.de/cdms/molecules). Among these, approximately 50 are classified as complex organic molecules (COMs), i.e., comprised of six atoms or more. COMs are generally observed in the gas phase, although it is widely accepted that saturated COMs are formed primarily through reactions on the surface of icy dust grains. This is where gas-phase species accrete, meet and react to form saturated molecules. One of the formation mechanisms that is often invoked is a diffusive mechanism or Langmuir-Hinshelwood (Cuppen et al. 2017). In this mechanism, species diffuse over surface to meet before they react. Whereas this mechanism, is efficient for hydrogenation reactions at low temperatures where atomic hydrogen diffuses over the surface (Hama et al. 2012; Kuwahata et al. 2015) and reacts with more-or-less stationary species, the mechanism cannot explain the formation of more complex species where radicals that are more strongly bound need to diffuse. Take methane as an example. This is a moderately sticky molecule and many species will have binding energies roughly in the same ball park. According to Smith et al. (2016), its binding energy is $1370 \mathrm{~K}$. If we assume a diffusion-to-binding energy ratio of 0.35 (Karssemeijer \& Cuppen 2014), the hopping rate becomes around 45 hops per year

(c) The Author(s), 2020. Published by Cambridge University Press on behalf of International Astronomical Union. This is an Open Access article, distributed under the terms of the Creative Commons Attribution-NonCommercial-NoDerivatives licence (http://creativecommons.org/licenses/by-nc-nd/4.0/), which permits non-commercial re-use, distribution, and reproduction in any medium, provided the original work is unaltered and is properly cited. The written permission of 
on a $10 \mathrm{~K}$ grain. This means that it takes $2 \times 10^{4}$ years for a single $\mathrm{CH}_{4}$ molecule to scan the surface of a grain and even longer to desorb. This is too slow to get chemistry at dense cloud conditions, especially for minority species.

COMs like $\mathrm{CH}_{3} \mathrm{CHO}, \mathrm{HCOOCH}_{3}$ and $\mathrm{CH}_{3} \mathrm{OCH}_{3}$ have however been detected in the gas phase of prestellar cores (Bacmann et al. 2012; Soma et al. 2018). These molecules are thought to form on grain surfaces through radical-radical recombination. The question is how can they form at these low temperatures where thermal diffusion is limited and how do they desorb to be detected in the gas phase. One possibility is that they can form without diffusion but through the formation of radicals in close vicinity of each other as was shown by Fedoseev et al. (2015). Another possibility is that the exothermicity of one reaction, for instance hydrogenation, can induce chemical or non-thermal diffusion, where the energy released during the surface reaction allows the product to diffuse over the surface with the possibility to meet another reactant for subsequent reactions (Arasa et al. 2010; Lamberts et al. 2014). In this way, the excess energy of one reaction would enable subsequent reactions. The exothermicity can also be applied for chemical or reactive desorption to return species to the gas phase.

Here we present Molecular Dynamics simulations to examine the role of exothermicity in non-thermal desorption and diffusion and to quantify the outcome of exothermicity. The excitation energy can be applied for diffusion and desorption, but also for restructuring of the grain mantle. The final aim of the work is to obtain general formalisms to describe desorption probability and/or thermal diffusion that can be used in astrochemical gas-grain models.

\section{Molecular Dynamics set-up}

Molecular Dynamics (MD) simulations follow Newton's equations of motion and allow one to keep track of the energy, potential or kinetic energy, by giving a species some initial energy. Here we aim to investigate the fate of excited species on the surface of an ice mantle. Excess energy can be in the form of translational, rotational, or vibrational energy or electronic excitation. How this energy is spread is highly system dependent(Polanyi 1986); it does not only depend on the specific reaction but also on the reaction environment and the configuration in which it occurs. Initially, we only consider an additional kinetic energy, but we will also consider vibrational and rotational excitation. A single Molecular Dynamics simulation can follow a single excitation event. To understand how excess energy can influence desorption and diffusion, we need to perform many individual simulations with slightly different initial conditions and determine the distribution over the different outcomes. This further needs to be repeated for many different excitation energies.

Here we choose a kinetic energy range between 0.5 and $5 \mathrm{eV}$ to cover the full range from reactions with a small exothermicity to photodissociation reactions where the excitation energy after bond breaking can be several eV. Both crystalline and amorphous water ice are used as a substrate and three different surface species are considered, $\mathrm{CO}_{2}, \mathrm{H}_{2} \mathrm{O}$ and $\mathrm{CH}_{4}$, which are among the most common ice species (Boogert et al. 2015). Moreover, these species span a range in binding energies, number of internal degrees of freedom and molecular weight.

We roughly apply the following procedure: First the hundreds of deposition simulations are issued where the admolecules can find the different binding sites on the substrate. Next, the strongest binding sites are selected, and from each site the admolecule is given a specific amount of additional translational kinetic energy in a random direction. Finally, the outcome of the simulation is detected by the software and stored for post-analysis. We classify each simulation into: desorption, adsorption, penetration, FAIL, and TIME 
LIMIT. For more details, we refer to Fredon et al. (2017); Fredon \& Cuppen (2018). For each kinetic energy value 1,400 individual simulations are performed.

\section{Results}

\subsection{Crystalline vs. amorphous substrates}

For all three admolecules, desorption or adsorption are the most likely outcomes; penetration is found to occur in only a few simulations, all for high kicking energies. Figure 1 shows the distribution of these outcomes as a function of initial kinetic energy. Panel (a) is for admolecules on a crystalline surface. What is apparent from these graphs is that the desorption probability increases with kinetic energy. Moreover, $\mathrm{CH}_{4}$ desorbs more easily than $\mathrm{CO}_{2}$ and $\mathrm{H}_{2} \mathrm{O}$, respectively, which is in line with their binding energies to a water surface. For low excitation energy, there is an onset for a desorption. If the kinetic energy is lower than the binding energy, the admolecules cannot desorb.

Comparison with the results on an ASW surface (panel b) shows two clear differences: penetration occurs more often and the asymptotic desorption probability for high excitation energies does not go to one, but to a lower value, especially for methane. On close inspection of the corresponding trajectories, it becomes clear that this is due to the adsorption depth of the admolecules to the surface. During deposition, the admolecules really stay on the surface for the crystalline surface, whereas they can slowly accommodate into a surface pore on ASW. Water molecules that approach the surface form hydrogen bonds as soon as they reach the surface, resulting in a rather low adsorption depth. $\mathrm{CH}_{4}$ molecules on the other hand can roam the surface until they find a pore. The adsorption depth of $\mathrm{CO}_{2}$ is between these two extremes. Once in a pore diffusion and adsorption is much harder and hence the traveled distance and the desorption probability reduces with respect to a crystalline surface.

If we consider the applied kinetic excitation as a proxy for reaction excess energy in translational excitation $E_{\text {react }}^{\text {trans }}$, the chemical desorption probability is described with the following expression

$$
P_{\mathrm{CD}}=f\left[1-\exp \left(-\omega \frac{E_{\text {react }}^{\mathrm{trans}}-\left|E_{\mathrm{bind}}\right|}{\left|E_{\mathrm{bind}}\right|}\right)\right]
$$

with $E_{\text {bind }}$ the binding energy to the surface and $\omega=0.3$ and $f$ two empirical parameters. The parameter $f$ depends on the adsorption depth of the species to the surface and the $\omega$ factor can be rationalized by the fact that only $z$-movement leads to desorption from the surface. Comparison with simulation results and other expressions for chemical desorption, proof of the threshold energy, and examples on how to apply the equation can be found in Fredon et al. (2017) and Fredon \& Cuppen (2018)

\subsection{The role of vibrational and rotational excitation}

Finally, we investigated the role of vibrational and rotational excitation to the desorption probability. Vibrational excitation has little-to-no effect on the desorption probability, whereas rotational energy can lower the threshold for desorption. The latter depends on the anisotropy of the molecule. The effect is largest for $\mathrm{CO}_{2}$ and no effect is visible for $\mathrm{CH}_{4}$. In general, a slow coupling between the different types of excitation is observed. Although this is not surprising, several expressions in the astrochemical literature for chemical desorption assume equipartition (Garrod et al. 2007; Minissale et al. 2016). These expressions can also not reproduce our simulation results with one universal expression. 

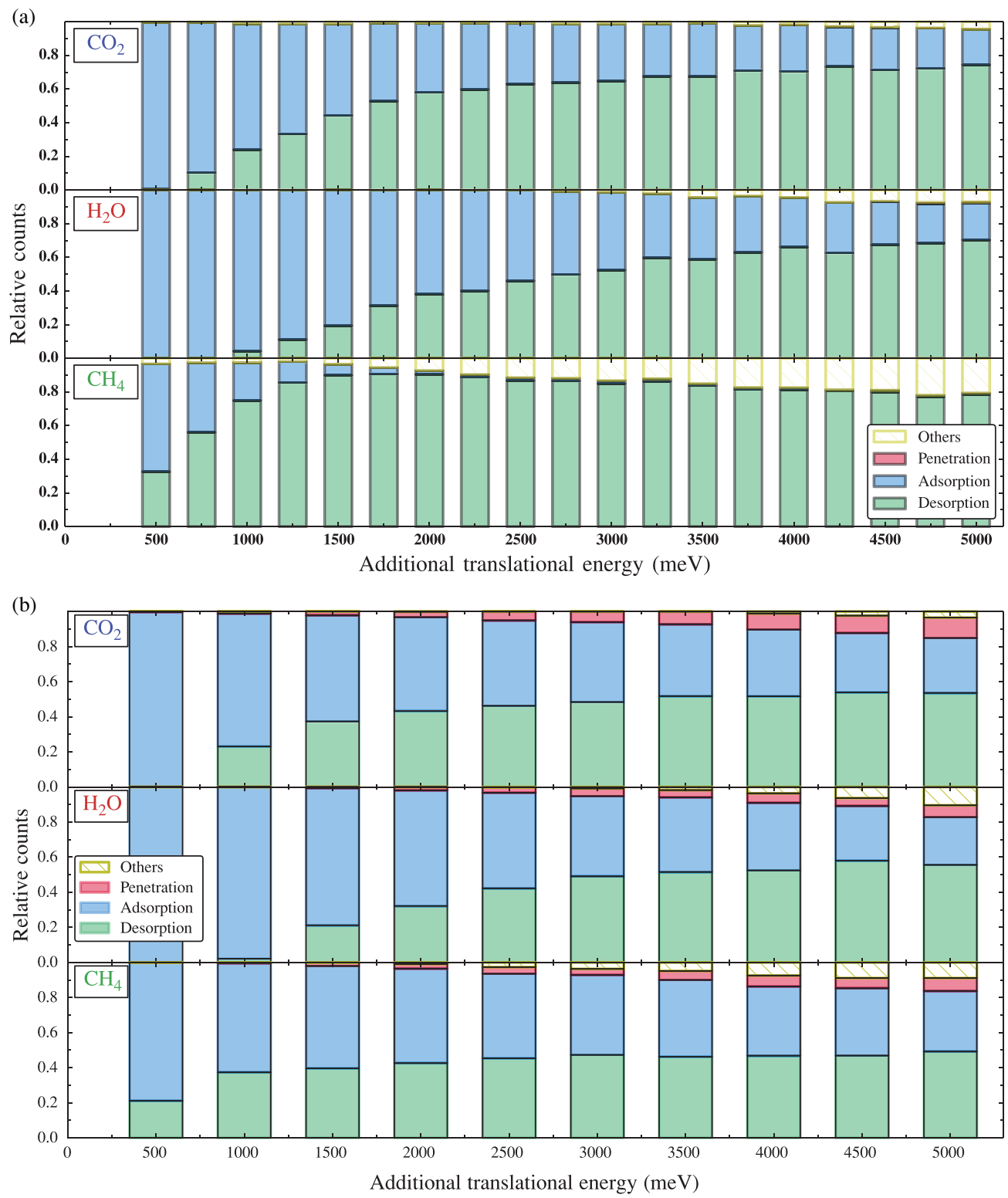

Figure 1. Distribution of the different outcomes as a function of the initial kinetic energy of the admolecule on (a) a crystalline surface and (b) an amorphous water ice surface. Figure reproduced from (a) Fredon et al. (2017) and (b) Fredon \& Cuppen (2018).

\section{Conclusions}

In summary, we performed Molecular Dynamics simulations to study the role on excitation energy on diffusion and desorption. We found that molecules can scan a large part of the surface due to this excitation (not shown here). Chemical desorption is predominantly determined by the excess energy released in translational excitation. Rotational excitation plays a minor role. We hence recommend using Eq. 3.1 to describe the probability for chemical desorption. For the empirical parameters, we recommend $\omega=0.3$ and $f=0.5-0.8$. The main uncertainty remains $E_{\text {react }}^{\text {trans }} / E_{\text {react }}$. We expect translational 
and rotational energy to play an important role for reactions with bond breaking, like abstraction reactions and photodissociation reactions, whereas in association reactions vibrational excitation is most likely the most dominant dissipation channel.

\section{Acknowledgment}

AF and HMC acknowledge NWO for financial support (ECHO 712.014.004).

\section{References}

Arasa, C., Andersson, S., Cuppen, H. M., van Dishoeck, E. F., \& Kroes, G. 2010, J. Chem. Phys. 132(18), 184510

Bacmann, A., Taquet, V., Faure, A., Kahane, C., \& Ceccarelli, C. 2012, A\&A, 541, L12

Boogert, A. A., Gerakines, P. A., \& Whittet, D. C. 2015, ARA\&SA, 53, 541

Cuppen, H. M., Walsh, C., Lamberts, T., Semenov, D., Garrod, R. T., Penteado, E. M., \& Ioppolo, S. 2017, Space Sci. Rev. 212, 1

Fedoseev, G., Cuppen, H. M., Ioppolo, S., Lamberts, T., \& Linnartz, H. 2015, MNRAS 448, 1288

Fredon, A. \& Cuppen, H. M. 2018, PCCP, 20, 5569

Fredon, A., Lamberts, T., \& Cuppen, H. M. 2017, ApJ 849, 125

Garrod, R. T., Wakelam, V., \& Herbst, E. 2007, A\&广A 467, 1103

Hama, T., Kuwahata, K., Watanabe, N., Kouchi, A., Kimura, Y., Chigai, T., \& Pirronello, V. 2012, ApJ 757, 185

Karssemeijer, L. J. \& Cuppen, H. M. 2014, A\&A 569, A107

Kuwahata, K., Hama, T., Kouchi, A., \& Watanabe, N. 2015, Phys. Rev. Lett. 115, 133201

Lamberts, T., de Vries, X., \& Cuppen, H. M. 2014, Faraday Disc. 168, 327

Minissale, M., Dulieu, F., Cazaux, S., \& Hocuk, S. 2016, A\&3A, 585, A24

Polanyi, J. C. 1986, Noble Lecture Chemistry

Smith, R. S., May, R. A., \& Kay, B. D. 2016, J. Phys. Chem. B 120, 1979

Soma, T., Sakai, N., Watanabe, Y., \& Yamamoto, S. 2018, ApJ, 854, 116 
